\title{
Reconstitution dendrochronologique et fréquence des grosses avalanches de neige dans un couloir subalpin du mont Hog's Back, en Gaspésie centrale (Québec) Tree-Ring Reconstruction and Frequency of High-Magnitude Snow Avalanches in a Subalpine Avalanche Track, Mont Hog's Back, Central Gaspésie (Québec) Reconstitución dendrocronológica y frecuencia de avalanchas importantes en el corredor subalpino del Mont Hog's Back, en Gaspésie central (Québec)
}

Dominic Boucher, Louise Filion et Bernard Hétu

Volume 57, numéro 2-3, 2003

URI : https://id.erudit.org/iderudit/011311ar

DOI : https://doi.org/10.7202/011311ar

\section{Aller au sommaire du numéro}

\section{Éditeur(s)}

Les Presses de l'Université de Montréal

\section{ISSN}

0705-7199 (imprimé)

1492-143X (numérique)

\section{Découvrir la revue}

\section{Citer cet article}

Boucher, D., Filion, L. \& Hétu, B. (2003). Reconstitution dendrochronologique et fréquence des grosses avalanches de neige dans un couloir subalpin du mont Hog’s Back, en Gaspésie centrale (Québec). Géographie physique et Quaternaire, 57(2-3), 159-168. https://doi.org/10.7202/011311ar

\section{Résumé de l'article}

Cette étude porte sur l'activité des grosses avalanches de neige dans un couloir de $660 \mathrm{~m}$ de longueur, sur le versant est du mont Hog’s Back, en Gaspésie centrale (Québec). L’analyse dendrochronologique des arbres échantillonnés dans la zone d'arrivée des coulées de neige a permis de faire ressortir 35 événements survenus entre 1895 et 1996, ce qui correspond à un intervalle de retour des grosses avalanches de près de 3 ans et à une fréquence ou probabilité d'incidence annuelle d'environ $34 \%$. Les décennies 1950 et 1980 rassemblent le plus grand nombre d'événements, respectivement 5 et 6 grosses avalanches, et la décennie 1930, aucun. La probabilité annuelle d'incidence de grosses avalanches dans le couloir étudié a été évaluée à $27 \%$ et $41 \%$ respectivement pour la première (1895-1945) et la seconde (1946-1996) parties $\mathrm{du} \mathrm{XX} \mathrm{X}^{\mathrm{e}}$ siècle. Les données sur les précipitations de neige obtenues d'une station (Cap-Seize) située à proximité du couloir étudié montrent qu’entre 1969 et 1994, 8 des 13 années de grosses avalanches (62 \%) ont enregistré des précipitations totales de neige supérieures à la moyenne $(457 \mathrm{~cm})$. Les facteurs d'ordres topographique, écologique et climatique favorisant le déclenchement d’avalanches en Gaspésie centrale sont aussi abordés. 


\section{RECONSTITUTION}

DENDROCHRONOLOGIQUE

ET FRÉQUENCE DES GROSSES AVALANCHES DE NEIGE DANS UN COULOIR SUBALPIN DU MONT HOG'S BACK, GASPÉSIE CENTRALE (QUÉBEC)

Dominic BOUCHER ${ }^{\star}$, Louise FILION et Bernard HÉTU, premier et deuxième auteurs : Département de géographie et Centre d'études nordiques, Université Laval, Québec G1K 7P4 ; troisième auteur : Module de géographie et Centre d'études nordiques, Université du Québec à Rimouski, 300, allée des Ursulines, Rimouski, Québec G5L 3A1.

RÉSUMÉ Cette étude porte sur l'activité des grosses avalanches de neige dans un couloir de $660 \mathrm{~m}$ de longueur, sur le versant est du mont Hog's Back, en Gaspésie centrale (Québec). L'analyse dendrochronologique des arbres échantillonnés dans la zone d'arrivée des coulées de neige a permis de faire ressortir 35 événements survenus entre 1895 et 1996, ce qui correspond à un intervalle de retour des grosses avalanches de près de 3 ans et à une fréquence ou probabilité d'incidence annuelle d'environ $34 \%$. Les décennies 1950 et 1980 rassemblent le plus grand nombre d'événements, respectivement 5 et 6 grosses avalanches, et la décennie 1930, aucun. La probabilité annuelle d'incidence de grosses avalanches dans le couloir étudié a été évaluée à $27 \%$ et $41 \%$ respectivement pour la première (1895-1945) et la seconde (1946-1996) parties du XXe siècle. Les données sur les précipitations de neige obtenues d'une station (Cap-Seize) située à proximité du couloir étudié montrent qu'entre 1969 et 1994, 8 des 13 années de grosses avalanches (62\%) ont enregistré des précipitations totales de neige supérieures à la moyenne $(457 \mathrm{~cm})$. Les facteurs d'ordres topographique, écologique et climatique favorisant le déclenchement d'avalanches en Gaspésie centrale sont aussi abordés.
ABSTRACT Tree-ring reconstruction and frequency of high-magnitude snow avalanches in a subalpine avalanche track, Mont Hog's Back, central Gaspésie (Québec). Highmagnitude snow avalanche activity was studied in a 660-m long avalanche track on the east slope of Mont Hog's Back in central Gaspésie (Québec). Tree-ring data from trees growing in the runout zone, which were damaged during snow wasting events, indicated that 35 high-magnitude avalanches occurred between 1895 and 1996, which corresponds to a $\sim 3$-year return interval with a $\sim 34 \%$ probability that an avalanche will occur in any one year. The highest number of events was recorded in the 1950s and the 1980s, with 5 and 6 events, respectively, whereas no avalanche was recorded in the 1930s. A $27 \%$ and $41 \%$ annual frequency or probability of high-magnitude avalanches in the avalanche track studied was calculated for the first (1895-1945) and the second part (1946-1996) of the 20th century. Snowfall from a nearby meteorological station (CapSeize) indicated that 8 out the 13 high-magnitude avalanche years (62\%) between 1969 and 1994 had total snowfall above average $(457 \mathrm{~cm})$. Geographical, ecological and meteorological factors responsible for avalanche activity in central Gaspésie were also discussed.
RESUMEN Reconstitución dendrocronológica y frecuencia de avalanchas importantes en el corredor subalpino del Mont Hog's Back, en Gaspésie central (Québec). El presente estudio trata de la actividad de las avalanchas importantes en un corredor de $600 \mathrm{~m}$ de largo, en la vertiente este del Mont Hog's Back, en la región de la Gaspésie central (Québec). El análisis dendrocronológico de los árboles seleccionados en la zona donde atraviesan las caídas de nieve permitió identificar 35 eventos acontecidos entre 1895 y 1996, que corresponden a intervalos de ocurrencia de avalanchas importantes de cerca de 3 años y de una frecuencia o probabilidad de acontecimiento anual cercana al $34 \%$. Las décadas de 1950 y 1980 reúnen el mayor número de ocurrencia de grandes avalanchas, 5 y 6 respectivamente; mientras que la década de 1930 no registra ninguna. La probabilidad anual de ocurrencia de grandes avalanchas en el corredor estudiado fue estimada entre 27 y $41 \%$ para la primera y la segunda parte del siglo XX, 1895-1945 y 1946-1996 respectivamente. Los registros de caída de nieve obtenidos de la estación de Cap-Seize, situada a proximidad del corredor subalpino, muestran que entre 1969 y 1994, 8 de los 13 años que registran avalanchas importantes $(62 \%)$ poseen un nivel de caída de nieve total superior al promedio $(457 \mathrm{~cm})$. En el presente manuscrito se discuten también los factores de tipo topográfico, ecológico y climático que favorecen la formación de avalanchas en la región de la Gaspésie central.

Manuscrit reçu le 2 juillet 2002 ; manuscrit révisé accepté le 26 novembre 2003 (publié le $3^{e}$ trimestre 2005)

*Adresse actuelle : Centre d'avalanche de la Haute-Gaspésie, 464, boul. Sainte-Anne ouest, Sainte-Anne-des-Monts, Québec G4V 1 T5.

Adresse électronique : dominic@ centreavalanche.qc.ca 


\section{INTRODUCTION}

Depuis le début des années 1970, les avalanches de neige ont causé la mort de plus d'une trentaine de personnes au Québec (Stethem et Schaerer, 1979, 1980; Schaerer, 1987; Hétu et Dubé, 1995, 1999 ; Jamieson et Brooks, 1998). Compte tenu de son relief accidenté et des grandes quantités de neige qu'elle reçoit, la Gaspésie constitue une région propice aux avalanches, en particulier sur les pentes surmontées d'un étage alpin. II semble que le niveau de risque soit en croissance rapide à cause de l'augmentation continuelle du nombre de personnes s'adonnant au ski hors-piste (Boucher, 2000). Bien que les avalanches soient connues depuis longtemps en Gaspésie (Gaumond et Hamelin, 1960), ce n'est que récemment que des recherches visant à en préciser la répartition et la fréquence ont commencé. Outre ceux identifiés par la cartographie régionale à petite échelle produite par Veillette et Cloutier (1993), les secteurs les mieux connus en Gaspésie sont les régions de Mont-Saint-Pierre (Hétu et Vandelac, 1989; Dubé, 1999; Hétu et Gray, 2000) et du mont Albert (Girard et Hétu, 1989, 1994; Larocque et al., 2001) où des travaux portant sur les impacts à la fois géomorphologiques et dendroécologiques des avalanches et des coulées de neige liquéfiée (slushflows) ont été récemment effectués. Mais beaucoup de travail reste à faire dans cette vaste région qui compte plus de 300 couloirs d'avalanche répartis sur l'ensemble du territoire (Veillette et Cloutier, 1993).

Cette étude a été effectuée en Gaspésie centrale, dans un couloir d'avalanche du mont Hog's Back, lequel est situé dans les monts Chic-Chocs, à quelque $40 \mathrm{~km}$ du littoral nord de la péninsule gaspésienne (fig. 1). La fréquentation de cette montagne par les skieurs hors-piste n'a vraisemblablement commencé qu'à I'hiver 1997-1998, soit depuis la mise en place d'un stationnement au pied du versant est, le plus actif en ce qui a trait aux avalanches. II semble que peu de skieurs fréquentent les couloirs d'avalanche sur ce versant et que la majorité d'entre eux empruntent plutôt le versant sud, de sorte que le régime d'avalanche étudié est encore naturel et ne découle pas d'une fréquentation accrue au cours des dernières années. Les objectifs de cette étude sont de reconstituer l'historique des avalanches de neige par une analyse dendrochronologique détaillée des arbres endommagés dans ce couloir et de déterminer la fréquence des grosses avalanches de neige, soit celles qui se rendent jusqu'à la forêt de conifères occupant le pied du versant. De nombreux auteurs ont employé une telle méthode, surtout dans les Rocheuses canadiennes et américaines (Burrows et Burrows, 1976; Shroder, 1978; Carrara, 1979; Niemann, 1982; Butler et Malanson, 1985a, 1985b; Johnson et al., 1985; Veblen et al., 1994; Rayback, 1998) pour reconstituer l'historique des avalanches mais aussi pour calculer l'intervalle de retour des grosses avalanches, soit l'intervalle de temps moyen entre deux avalanches de grande amplitude, ainsi que leur fréquence ou probabilité annuelle d'incidence (Potter, 1969; Shroder, 1978, 1980; Johnson, 1987; Patten et Knight, 1994; Larocque et al., 2001). Les données récoltées dans le cadre de cette étude ont non seulement permis de préciser la fréquence et l'intervalle de retour des avalanches dans le couloir étudié, mais également de mettre en évidence les changements de régime au cours du dernier siècle.

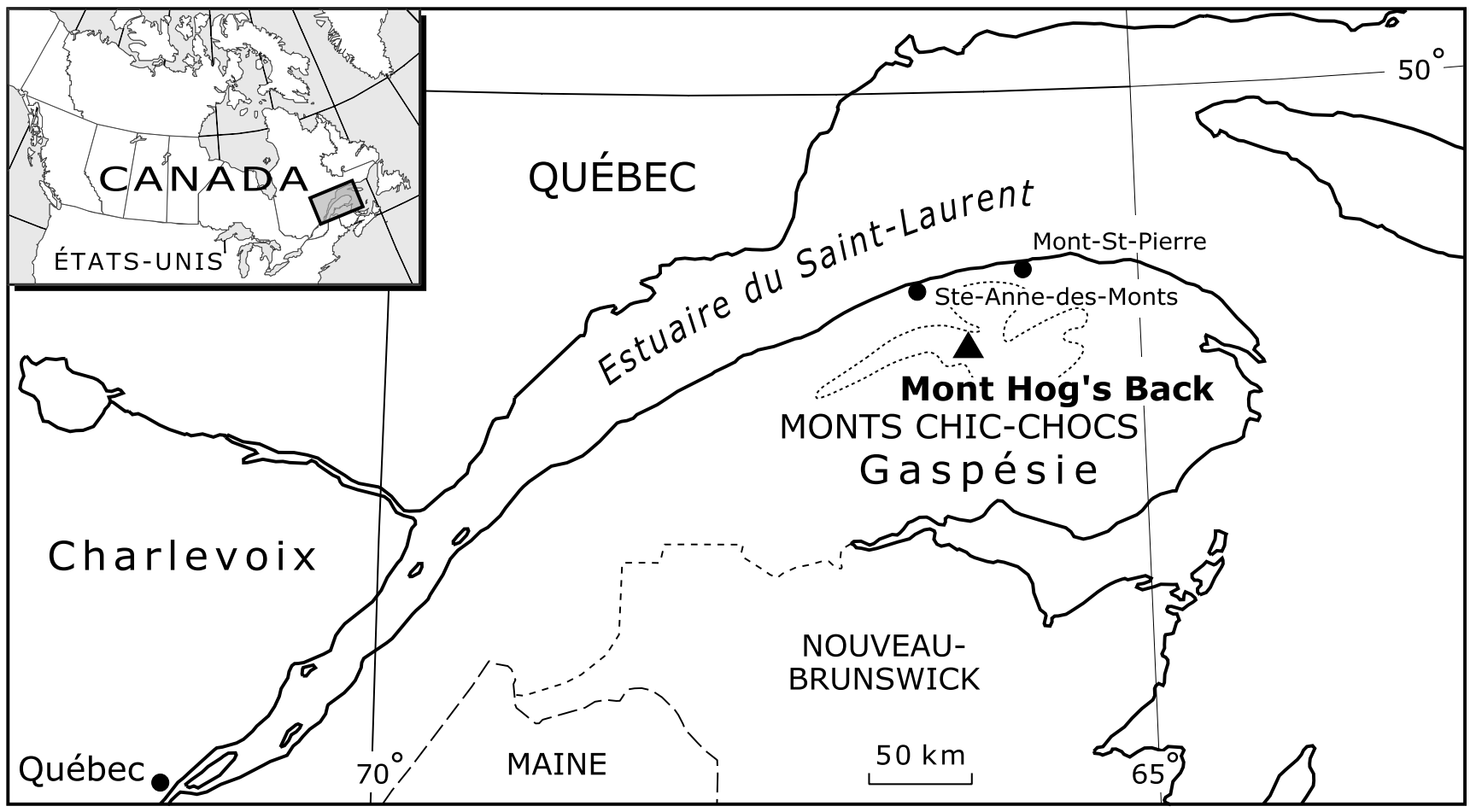

FIGURE 1. Localisation de la région et du site à l'étude.

Location of the study area and site. 
Les données météorologiques sur le secteur de la Gaspésie centrale sont peu nombreuses, mais nous avons pu obtenir celles portant sur les précipitations de neige enregistrées à la station du Cap-Seize, située à 229 m d'altitude entre SainteAnne-des-Monts et le mont Hog's Back, à quelque $30 \mathrm{~km}$ au nord du couloir d'avalanche étudié (fig. 1). Ces données ont permis d'évaluer le lien entre la quantité de neige tombée au sol et l'incidence des grosses avalanches de neige.

\section{RÉGION ET SITE À L'ÉTUDE}

Culminant vers 830 m d'altitude, le mont Hog's Back est situé au sein des monts Chic-Chocs (fig. 1). Cette montagne à la crête étroite, bordée de versants raides (fig. 2A), correspond à une intrusion granitique dont la mise en place remonte au Dévonien (Doyon et Valiquette, 1991; Brisebois et Brun, 1994). La température moyenne annuelle passe de $+0,3^{\circ} \mathrm{C}$ vers $550 \mathrm{~m}$ d'altitude à $-4^{\circ} \mathrm{C}$ vers 1 100-1 $200 \mathrm{~m}$ (Gray et Brown, 1979, 1982). Avec des précipitations annuelles qui varient entre 1100 et $1600 \mathrm{~mm}$, dont $40 \%$ tombent sous forme de neige, et une nébulosité moyenne qui dépasse les $70 \%$, les Chic-Chocs comptent parmi les régions les plus humides du Québec (Gagnon, 1970).

Le versant est du mont Hog's Back présente deux couloirs d'avalanche bien développés dans lesquels on distingue clairement les zones de départ, de transfert et d'arrivée des grosses avalanches (fig. 2B). Sous les $600 \mathrm{~m}$ d'altitude, ces couloirs sont clairement délimités par la forêt qui présente une trimline. Le couloir nord, étudié ici, s'étire sur plus de $660 \mathrm{~m}$ de longueur et sa partie distale, en vue verticale, présente une forme bilobée (fig. 2C). Située dans l'étage alpin, la zone de départ occupe un petit bassin versant en forme d'entonnoir qui se déploie entre 600 et $800 \mathrm{~m}$ d'altitude. Les pentes, dont l'inclinaison varie entre $34^{\circ}$ et $36^{\circ}$, y sont déboisées, mais quelques krummholz bas $(<2,5 \mathrm{~m})$ d'épinettes noires réussissent à s'y maintenir (fig. 2D). La zone de transfert, entre 530 et $600 \mathrm{~m}$ d'altitude, où l'inclinaison est d'environ $30^{\circ}$ et où croissent des krummholz bas mais aussi quelques krummholz hauts (2,5-5 $\mathrm{m}$ ) d'épinettes noires, est traversée par un ravin. Enfin, dans la zone d'arrivée, entre 450 et $530 \mathrm{~m}$ d'altitude et sur une pente variant de $22^{\circ}$ à $28^{\circ}$, des krummholz hauts (2,5-5 $\mathrm{m})$ d'épinettes noires et de sapins baumiers croissent en bordure de la forêt jusqu'ici épargnée par l'arrivée de masses de neige (fig. 2D). La végétation ligneuse dans les zones de transfert et d'arrivée présente plusieurs types de dommages (troncs cassés, cicatrices d'impact, élagage des branches dans la partie amont) et de nombreuses tiges sont inclinées vers le bas de la pente, ce qui témoigne du passage récent et récurrent de coulées de neige.

\section{MÉTHODES}

\section{ÉCHANTILLONNAGE}

Le couloir d'avalanche nord a été préféré au couloir sud en raison de son potentiel dendrochronologique plus élevé dans la zone d'arrivée. Comme le couloir sud est plus long, sa zone d'arrivée a été grandement perturbée par la construction d'une ligne de transport électrique (fig. 2B). Un profil longitudinal du versant a été levé au centre du couloir nord à l'aide d'une boussole Brunton (précision : $\pm 1^{\circ}$ ). Les principales unités de végétation dans le couloir d'avalanche ont été sommairement décrites ainsi que la composition (espèces dominantes) de la forêt adjacente au couloir.

La surface du couloir d'avalanche à l'étude a été délimitée sur le terrain, ce qui a permis, grâce à un système de coordonnées, de localiser les trois transects d'échantillonnage ainsi que les arbres endommagés par les coulées de neige. Dans le couloir d'avalanche, 62 arbres vivants ont été échantillonnés sur une largeur de $2 \mathrm{~m}$ le long de trois transects transversaux, soit T1 $(n=30)$, T2 $(n=22)$ et T3 $(n=10)$, tous situés dans la zone d'arrivée des grosses avalanches, respectivement à 450, 460 et $475 \mathrm{~m}$ d'altitude (fig. $2 \mathrm{C}$ ). La position des transects sur le versant a été déterminée de façon à pouvoir répertorier le maximum d'arbres endommagés. Les anomalies ou dommages répertoriés ont été l'inclinaison de la tige principale entraînant le formation de bois de réaction, les cicatrices d'abrasion et les cassures de la tige principale. Des sections transversales ont été prélevées à tous les niveaux sur la tige où de tels dommages étaient apparents et, dans le cas des tiges inclinées, dans la courbure du tronc, là où se forme le bois de réaction en réponse à l'inclinaison de la tige. Certaines cassures ont pu être datées par interdatation dendrochronologique (cross dating), en comparant le patron de croissance de l'arbre touché à celui de ses voisins et par le recours aux cernes diagnostiques.

Dans la forêt bordant le couloir d'avalanche, des carottes ont été prélevées sur 52 arbres sains pour déterminer l'âge des plus vieux arbres dans le secteur adjacent à la zone d'arrivée des avalanches. Une seule carotte sur chaque arbre sélectionné parmi les plus gros (considérés comme étant les plus âgés) a été extraite, le plus près possible du collet $(<30 \mathrm{~cm})$, à l'aide d'une sonde de Pressler. Les arbres les plus âgés fournissent en effet un âge minimal d'établissement de la forêt à cette hauteur dans le versant (450-530 m).

\section{RECONSTITUTION DENDROCHRONOLOGIQUE ET FRÉQUENCE DES AVALANCHES}

Les échantillons récoltés ont été finement poncés et analysés en laboratoire afin de déterminer l'année d'inclinaison des troncs et de formation des dommages causés aux arbres, ainsi que l'âge des arbres échantillonnés dans le couloir d'avalanche et dans la forêt adjacente. Les cernes annuels de croissance ont été datés avec précision (résolution annuelle), grâce à la présence de certains cernes diagnostiques, en particulier les cernes étroits formés en 1978, 1979, 1984 et 1991. Les indicateurs retenus pour la reconstitution historique des avalanches sont les séquences de bois de réaction d'une durée minimale de deux ans, ainsi que les cassures du tronc et les cicatrices d'abrasion sur la tige principale. L'inventaire des séquences de bois de réaction et des dommages a été effectué pour chaque arbre. Puis, les données ont été compilées pour chacun des transects sous la forme d'un histogramme de fréquence pondérée des réponses annuelles (Shroder, 1978) afin d'identifier les années probables d'avalanche. Pour chaque année $t$, l'indice $I_{t}$ correspond au pourcentage de 

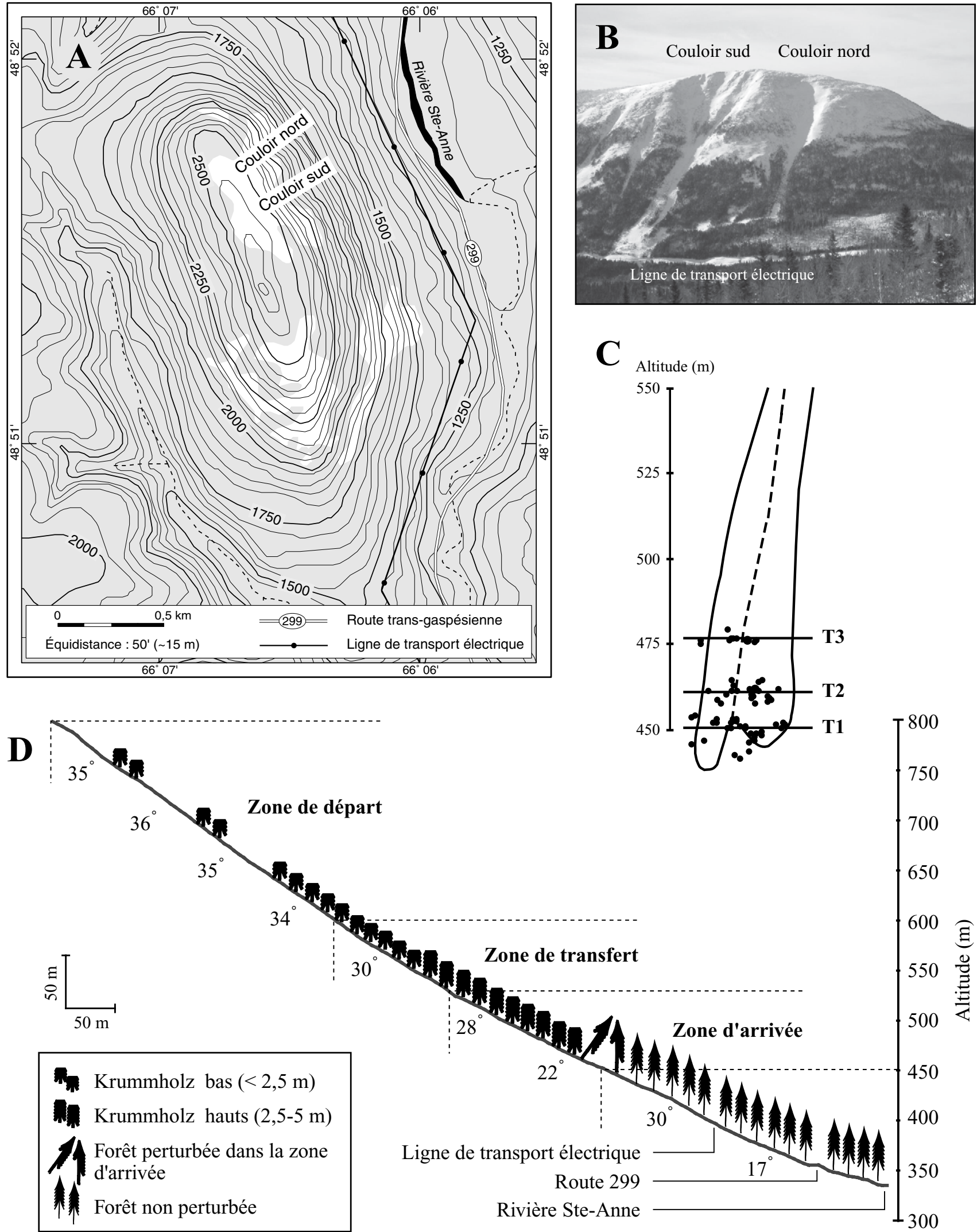

FIGURE 2. Topographie (A) et vue générale montrant deux couloirs d'avalanche sur le versant est du mont Hog's Back (B). En (C), la position des trois transects d'échantillonnage des arbres à des fins de reconstitution dendrochronologique des avalanches et en (D), le profil du versant ainsi que la végétation ligneuse dans le couloir d'avalanche nord.
Contour map (A) and general view of the eastern slope of Mont Hog's Back showing the position of two avalanche tracks $(B)$. In (C), the position of the three transects used for tree sampling in tree-ring reconstruction of avalanches and (D), slope long profile along with woody vegetation in the northern avalanche track. 
réponses obtenues $(R)$ en fonction du nombre d'arbres vivants $(V)$ cette même année $(t)$ :

$$
I_{t}=\left(\sum_{i=1}^{n} R_{t}\right) /\left(\sum_{i=1}^{n} V_{t}\right) \bullet 100
$$

À ce stade, les réponses obtenues du bois de réaction ont été distinguées de celles obtenues des dommages sur le tronc (cicatrices d'impact et cassures). Dans chaque transect, l'indice minimal retenu pour identifier les grosses avalanches de neige correspond à la moyenne des réponses obtenues pour la durée de chaque chronologie plus un écart type. Comme cet indice minimal change d'un transect à l'autre, il convient davantage aux échantillons de taille variable ( $n$ passant de 30 à 22 et 10 entre T1, T2 et T3), tout en étant suffisamment discriminant pour éliminer les années durant lesquelles les réponses sont trop faibles. II diffère du seuil de $10 \%$ utilisé par Bégin et Filion (1985) et Dubé et al. (2004) ou celui de $40 \%$ retenu par Butler et Malanson (1985b), ce dernier étant jugé trop élevé dans le contexte de notre étude. Nous avons fait débuter nos trois chronologies la même année (1895), soit la première année au cours de laquelle un événement a été enregistré avec un nombre d'arbres témoins $\geq 6$, dans l'un ou l'autre des transects, et selon le seuil retenu.

Les données obtenues des séquences de bois de réaction et des dommages aux tiges ont ensuite été compilées pour l'ensemble des trois transects, de façon à dresser la liste des années probables de grosses avalanches pour l'ensemble de la zone d'arrivée et ainsi calculer leur intervalle de retour $(r)$ depuis un siècle. L'intervalle de retour $r$ est obtenue en divisant une période donnée par le nombre d'avalanches enregistrées durant cette période. Leur fréquence ou probabilité annuelle, soit la fonction réciproque de leur intervalle de retour $(f=1 / r)$ exprimée en pourcentage, a aussi été calculée (Mears, 1992; McClung et Schaerer, 1993). Enfin, les arbres endommagés lors de chacune des grosses avalanches enregistrées par au moins 10 arbres témoins répartis dans au moins deux transects, ont été localisés précisément sur un fond cartographique pour déterminer leur extension spatiale à la base du couloir.

Les données sur les précipitations de neige en provenance de la station du Cap-Seize couvrent la période 1969 à 1994. La quantité totale de neige tombée au sol pendant un hiver donné (septembre d'une année à mai de l'année suivante) ainsi que par trimestre (septembre à novembre, décembre à février et mars à mai) a fait l'objet d'une analyse détaillée pour évaluer le lien entre l'incidence des grosses avalanches et les précipitations annuelles et saisonnières de neige, pour la période commune aux données météorologiques et dendrochronologiques. Enfin, il convient de mentionner que les années d'avalanche ont été identifiées par l'année comprenant les trois mois d'hiver (janvier à mars), ce qui revient à dire que l'année d'avalanche 1991, par exemple, correspond en fait à la saison hivernale 1990-1991.

\section{RÉSULTATS}

\section{HISTORIQUE DES GROSSES AVALANCHES DE NEIGE}

L'historique des avalanches de neige couvre la période comprise entre 1895 et 1996 (tabl. I). Bien que quelques tiges aient un âge assez avancé (âge maximal de 173 ans et âge moyen de 90 ans, $n=30$ ) le long du transect 1 situé près de la bordure forestière, elles étaient trop peu nombreuses pour permettre de couvrir adéquatement le XIXe siècle. Au total, 35 événements ont été enregistrés dans l'ensemble des transects pendant les 102 années couvertes par la chronologie des avalanches : 28 de ces années ont été identifiées grâce aux séquences de bois de réaction $(80 \%)$, dont $5(18 \%)$ par les deux types d'indicateurs, soit le bois de réaction ainsi que les dommages, et 7 autres (20\%) l'ont été uniquement par le biais des dommages causés aux tiges (cicatrices et cassures) (tabl. I et fig. 3). Les arbres échantillonnés le long des transects 1,2 et 3 ont permis d'enregistrer respectivement 19 (54\%), 17 (48\%) et 9 (26\%) des 35 événements (fig. 3), soit un nombre décroissant depuis la partie inférieure vers la partie supérieure de la zone d'arrivée des avalanches, tous indicateurs confondus (bois de réaction et dommages). Seulement trois années $(1969,1974$ et 1991) ont été enregistrées dans les trois transects.

La répartition des années d'avalanche par décennie fait ressortir un nombre inégal d'événements (tabl. I). Si l'on fait abstraction des deux décennies incomplètes (1890 et 1990), on obtient un nombre total de 28 événements répartis en neuf décennies, soit une moyenne de 3,11 avalanches par décennie. Les décennies 1950 et 1980 montrent le plus grand nombre d'événements et la décennie 1930 n'en comporte aucun. On obtient un intervalle moyen de retour $(r)$ de 2,91 pour toute la durée de la chronologie (102 ans), mais $r$ s'avère 1,5 fois plus long pour la première partie de la période considérée (1895-1945) que pour la seconde (tabl. II). La fréquence ou probabilité annuelle d'incidence de grosses avalanches

\section{TABLEAU I}

Années probables d'avalanche de neige et nombre d'événements par décennie entre 1895 et 1996, sur le versant est du mont Hog's Back, en Gaspésie centrale

\begin{tabular}{llc}
\hline Décennies & $\begin{array}{l}\text { Années probables d'avalanche à partir } \\
\text { des données dendrochronologiques }\end{array}$ & $\begin{array}{c}\text { Nombre } \\
\text { d'événements }^{2}\end{array}$ \\
\hline $1895-1899$ & $1895,1896,1898,1899$ & $4^{3}$ \\
$1900-1909$ & $1901,1903,1909$ & 3 \\
$1910-1919$ & 1914 & 1 \\
$1920-1929$ & $1921,1923,1926,1929$ & 4 \\
$1930-1939$ & nil & 0 \\
$1940-1949$ & $1941,1942,1948$ & 3 \\
$1950-1959$ & $1950,1951,1957,1958,1959$ & 5 \\
$1960-1969$ & 1964,1969 & 2 \\
$1970-1979$ & $1971,1974,1977,1978$ & 4 \\
$1980-1989$ & $1980,1981,1982,1985,1986,1987$ & 6 \\
$1990-1996$ & $1991,1993,1994$ & $3^{3}$ \\
\hline & Nombre total d'événements enregistrés & 35 \\
\hline
\end{tabular}

1. en italiques, les 7 années déduites uniquement à partir des dommages (cicatrices et cassures)

2. en caractères gras, les deux décennies avec le plus grand nombre d'avalanches de neige

3. nombre minimal obtenu à partir d'une décennie incomplète 

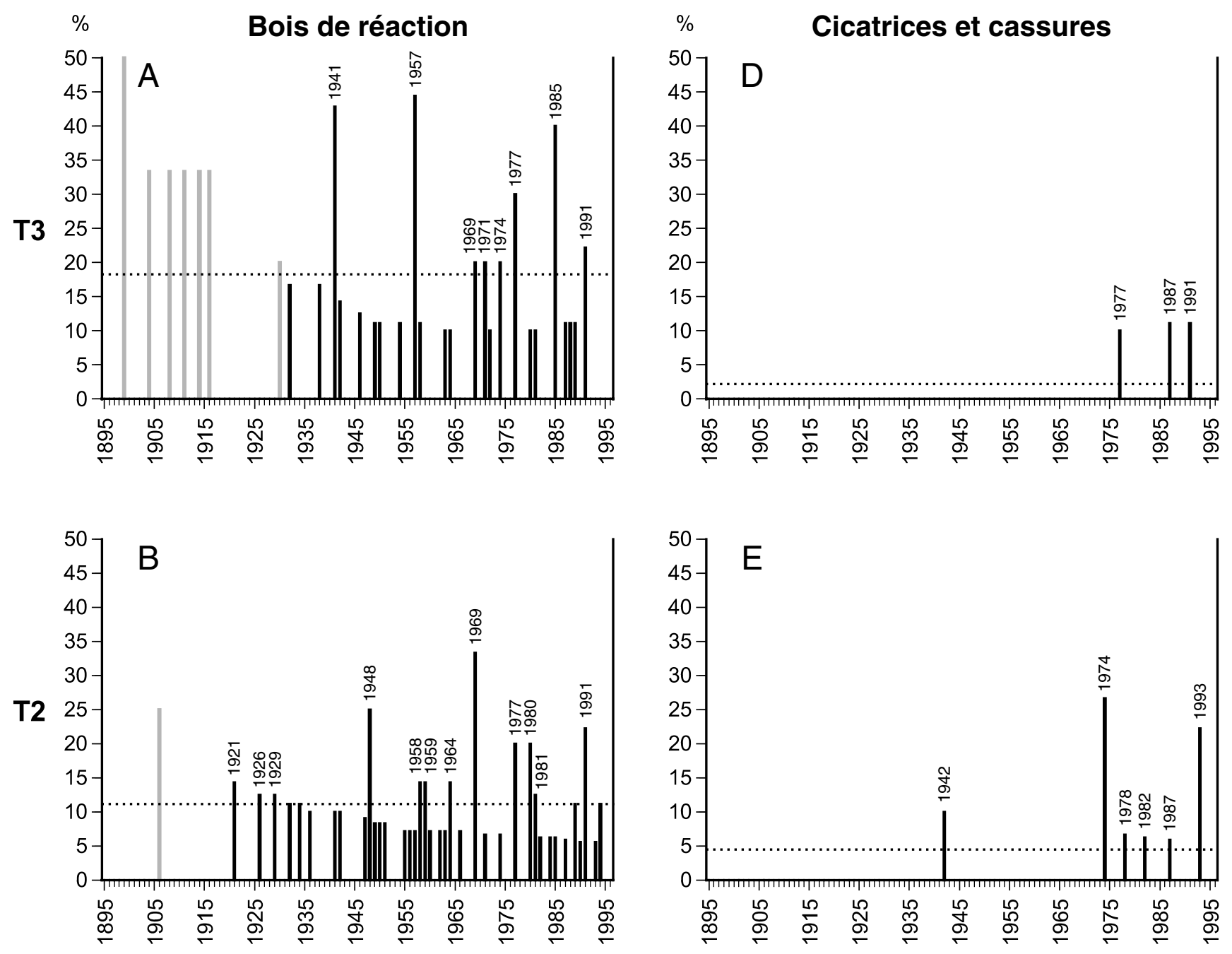

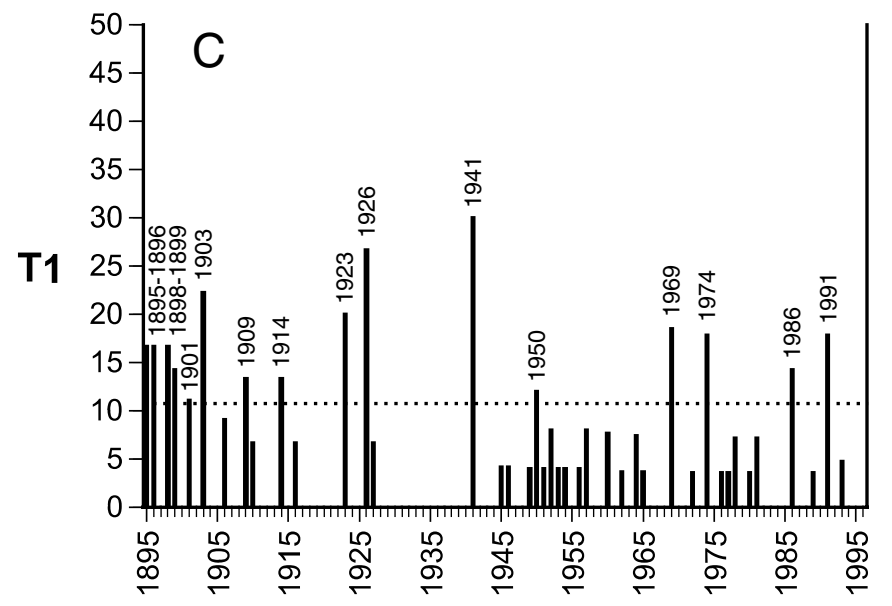

Années

FIGURE 3. Histogrammes des réponses pondérées obtenues à partir de l'analyse dendrochronologique du bois de réaction ( $A$ à $C$ ) et des dommages causés aux arbres échantillonnés (cicatrices d'impact et cassures de la tige principale) ( $\mathrm{D}$ à $\mathrm{F}$ ) dans chacun des trois transects (T1 à T3). Bâtonnets noirs : $n \geq 6$; bâtonnets gris $n<6$. Les traits horizontaux correspondent aux seuils de réponse calculés pour les divers transects et les types de dommages (voir la section Méthodes pour le calcul de ces seuils).

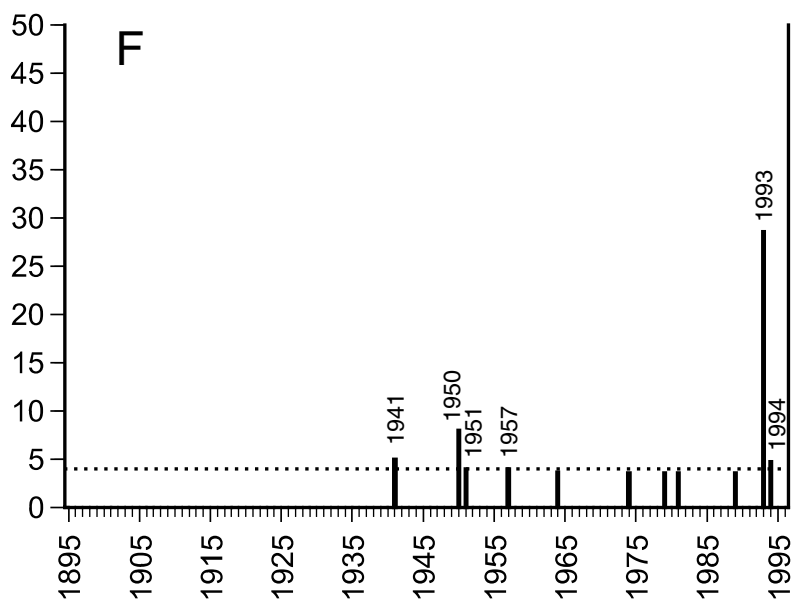

Années

Event-response histograms for inception of reaction wood (A to $C)$, impact scars and tree stem breakage ( $D$ to $F$ ) in each transect ( $T 1$ to T3). Black bars: $n \geq 6$; light bars : $n<6$. Horizontal dotted line $=\%$ threshold index calculated for each transect and type of damage (see Méthodes section for calculation of thresholds). 


\section{TABLEAU II}

Nombre d'années d'avalanche enregistrées, intervalle de retour ( $r$ ) et probabilité annuelle (p) d'incidence de grosses avalanches de neige pour les diverses périodes

\begin{tabular}{cccc}
\hline $\begin{array}{c}\text { Période } \\
\text { considérée }\end{array}$ & $\begin{array}{c}\text { Nombre d'années } \\
\text { d'avalanche }^{1}\end{array}$ & $\begin{array}{c}\text { Intervalle de retour } r \\
\text { en années }\end{array}$ & $\begin{array}{c}\text { Probabilité }(\%) \\
p=1 / r \times 100\end{array}$ \\
\hline $\begin{array}{c}1895-1996 \\
(102 \text { ans })\end{array}$ & 35 & 2,91 & 34 \\
$\begin{array}{c}1895-1945 \\
(51 \text { ans })\end{array}$ & 14 & 3,64 & 27 \\
$\begin{array}{c}1946-1996 \\
(51 \text { ans })\end{array}$ & 21 & 2,43 & 41 \\
\hline
\end{tabular}

1. Dans tous les cas, $n \geq 6$, soit le nombre minimal d'arbres témoins pour qu'un événement soit retenu.

sur le versant est du mont Hog's Back est de $34 \%$ sur la base des données séculaires et elle a été évaluée à $27 \%$ et $41 \%$, respectivement pour la première (1895-1945) et la seconde (1946-1996) partie du XXe siècle (tabl. II).

La cartographie des arbres endommagés lors des avalanches survenues en 1941, 1969, 1974, 1991 et 1993 révèle, dans certains cas, des trajectoires préférentielles dans le couloir (fig. 4). Alors que les avalanches survenues en 1941 et 1969 semblent avoir couvert l'ensemble de la zone d'arrivée, celles de 1974 et 1991 semblent plutôt centrées sur le secteur sud, et celle de 1993, sur le secteur nord. Cette dernière a, en plus, atteint la limite inférieure la plus basse dans le versant, vers $430 \mathrm{~m}$ d'altitude.

\section{RELATIONS ENTRE LES GROSSES AVALANCHES ET LES PRÉCIPITATIONS DE NEIGE}

Les données sur les précipitations de neige pour la période 1969-1994 (26 années) indiquent que les précipitations totales moyennes pour les 22 années pour lesquelles les données sont complètes ont été de $457,5 \mathrm{~cm}$ (tabl. III). Cette période comprend 14 des 35 années d'avalanche identifiées par les méthodes dendrochronologiques. Si l'on exclut l'année 1987 pour laquelle les données sont incomplètes, il demeure que des précipitations en neige supérieures aux précipitations moyennes ont été enregistrées pour 8 des 13 années de grosses avalanches (62\%). L'année 1991 est celle où on a enregistré le plus de neige $(757 \mathrm{~cm})$, soit 1,6 fois plus que la moyenne des 22 années pour lesquelles des données complètes sont disponibles. Les données trimestrielles indiquent que : 1) pendant chacun des trois trimestres considérés, les années 1977 et 1991 ont reçu plus de neige que la moyenne trimestrielle correspondante; 2) pendant le premier trimestre (septembre à novembre), l'année 1991 a reçu 3,6 fois plus de neige que la moyenne des 26 années; 3) pendant le second trimestre (décembre à février), l'année 1993 a reçu 1,8 fois plus de neige que la moyenne des 23 années pour lesquelles des données complètes sont disponibles et 4) pendant le troisième trimestre (mars à mai), les années 1971 et 1978 ont reçu environ 2 fois plus de neige que la moyenne des 26 années comprenant des données trimestrielles complètes. C'est plus précisément en novembre 1991 et en janvier 1993 que les écarts ont été les plus considérables.

\section{DISCUSSION}

Avec une probabilité annuelle d'avalanche de l'ordre de $41 \%$ depuis une cinquantaine d'années (entre 1946 et 1996), le versant est du mont Hog's Back est très propice aux avalanches de neige, les plus grosses correspondant à des décrochements de niveau 3 à 4 selon l'échelle d'intensité des avalanches qui comprend cinq niveaux (Canadian Avalanche Association, 1995). II s'agit d'une probabilité minimale puisque seules les grosses avalanches ont été considérées, soit celles qui se sont arrêtées dans la partie distale du couloir entre 450 et $530 \mathrm{~m}$ d'altitude. De nombreuses petites avalanches (niveau 1 à 2) peuvent en effet se produire plus haut dans le versant, sans laisser de traces visibles sur la végétation ligneuse, d'ailleurs clairsemée dans la zone de départ et de transfert.

Plusieurs facteurs d'ordres topographique, écologique et climatique favorisent le déclenchement spontané d'avalanches en Gaspésie centrale. Les valeurs de pente observées, de l'ordre de $34^{\circ}$ à $36^{\circ}$, se situent à l'intérieur de la fourchette caractéristique des pentes optimales $\left(30^{\circ}-40^{\circ}\right)$ pour la mise en mouvement des grosses avalanches à long intervalle de retour (Mears, 1992). Des chutes de neige abondantes totalisant 4 à $5 \mathrm{~m}$ par année, associées à une importante variabilité météorologique, entretiennent aussi des conditions d'instabilité sur

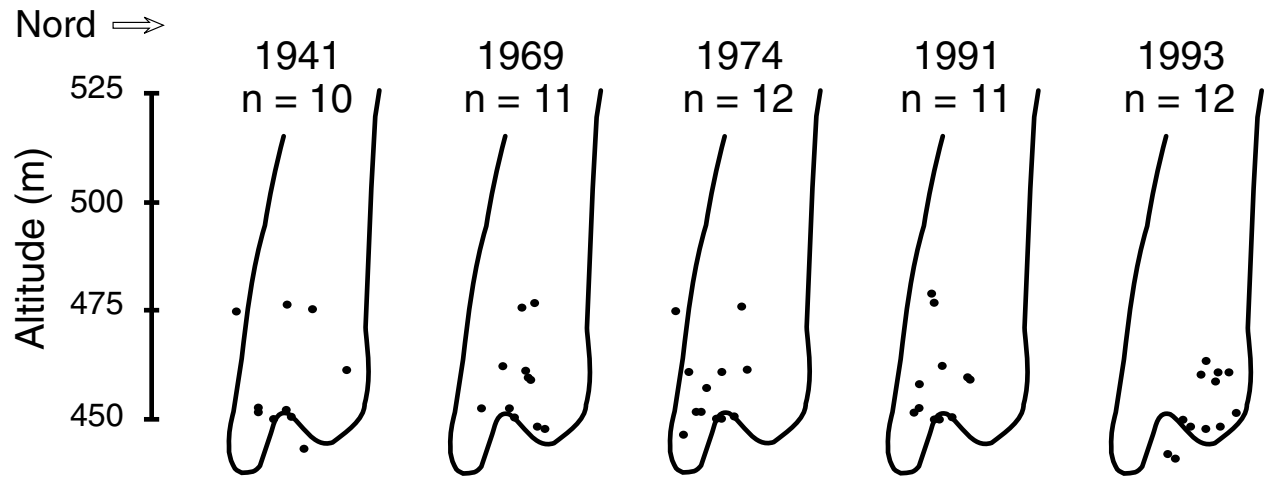

FIGURE 4. Répartition des arbres endommagés $(n \geq 10)$ lors des avalanches survenues en 1941, 1969, 1974, 1991 et 1993 dans le couloir d'avalanche nord.

Position of sampled trees $(n \geq 10)$ damaged during the 1941 1969-, 1974-, 1991- and 1993avalanche event in the northern avalanche track. 
TABLEAU III

Années probables d'avalanche pour chacun des transects, précipitations de neige par trimestre et précipitations totales annuelles, à la station du Cap-Seize (altitude : $229 \mathrm{~m}$ )

\begin{tabular}{|c|c|c|c|c|c|c|}
\hline \multicolumn{3}{|c|}{ Années d'avalanche par transect } & \multicolumn{4}{|c|}{ Précipitations de neige $(\mathrm{cm})$} \\
\hline $\mathrm{T}-3$ & $\mathrm{~T}-2$ & $\mathrm{~T}-1$ & $\begin{array}{c}\text { septembre à } \\
\text { novembre }\end{array}$ & $\begin{array}{l}\text { décembre à } \\
\text { février }\end{array}$ & $\begin{array}{c}\text { mars à } \\
\text { mai }\end{array}$ & Total $^{1}$ \\
\hline 1969 & 1969 & 1969 & 44 & 182,3 & 83,9 & 310,2 \\
\hline 1971 & & & 41 & 250,1 & 231,4 & 522,5 \\
\hline 1974 & 1974 & 1974 & 118,9 & 115,8 & 129,9 & 364,6 \\
\hline \multirow[t]{5}{*}{1977} & 1977 & & 87 & 355 & 170,2 & 612,2 \\
\hline & 1978 & & 24,8 & 284,4 & 232,3 & 541,5 \\
\hline & 1980 & & 41 & 387,3 & 100 & 528,3 \\
\hline & 1981 & & 115,4 & 250,7 & 124,6 & 490,7 \\
\hline & 1982 & & 43,9 & 319,3 & 104,2 & 467,4 \\
\hline \multirow[t]{2}{*}{1985} & & & 38,4 & 218 & 88,2 & 344,6 \\
\hline & & 1986 & 76,3 & 171,2 & 65,2 & 312,7 \\
\hline 1987 & 1987 & & 126,5 & $50^{2}$ & 48 & $224,5^{2}$ \\
\hline \multirow[t]{3}{*}{1991} & 1991 & 1991 & 268,2 & 281 & 208 & 757,2 \\
\hline & 1993 & 1993 & 64 & 485,8 & 89 & 638,8 \\
\hline & & 1994 & 70,5 & 213 & 145,4 & 428,9 \\
\hline
\end{tabular}

1. en caractères gras : années avec des précipitations totales supérieures à la moyenne

2. données incomplètes en décembre et pour le total annuel

3. moyenne des 22 années avec données complètes entre 1969 et 1994 (26 ans)

ces versants en pente raide (Gagnon, 1970; Hétu et Vandelac, 1989). La végétation ouverte qui caractérise les étages alpin et subalpin dans ce secteur de la Gaspésie centrale favorise en outre les transports nivéo-éoliens. Exposé à l'est, le versant étudié est en effet orienté sous le vent par rapport aux vents hivernaux efficaces, en particulier les blizzards associés au passage des masses d'air polaires. De grandes quantités de neige sont alors soufflées du flanc ouest de la montagne vers le haut du couloir d'avalanche, alimentant des corniches et des plaques à vent instables dont la rupture engendre des avalanches en plaque (slab avalanches) (ANENA, 1996; Jamieson et Geldsetzer, 1997).

Le manque de données météorologiques de longue durée à proximité du couloir étudié limite grandement l'analyse des facteurs météorologiques, locaux et régionaux responsables du déclenchement des avalanches. Cependant, la quantité de neige tombée au sol semble un facteur déterminant car, comme l'ont récemment montré Dubé et al. (2004) pour la frange maritime du nord de la Gaspésie, de nombreuses années d'avalanche identifiées correspondent à des hivers qui se distinguent par des précipitations de neige largement supérieures à la moyenne. Pour la période comprise entre 1939 et 1997 (59 ans), Dubé et al. ont obtenu une probabilité annuelle de 7 à $20 \%$ dans trois sites d'avalanche situés en contrebas de talus d'éboulis. Avec une probabilité annuelle de $41 \%$ - calculée pour la période allant de 1946 à 1996 (51 ans) qui est commune aux deux secteurs - le milieu subalpin du mont Hog's Back présenterait des risques d'avalanches de 2 à
6 fois plus élevés que le milieu maritime (région de Mont-SaintPierre). Par ailleurs, deux des six années de grosses avalanches identifiées par Dubé et al. en milieu maritime (1898, 1952, 1956, 1966, 1977, 1996) ont aussi été enregistrées en milieu subalpin (1898 et 1977). Deux d'entre elles (1952 et 1956) s'inscrivent dans une décennie au cours de laquelle la fréquence des avalanches au mont Hog's Back est élevée (1950-1960), bien que les années diffèrent. Il semble donc que des facteurs régionaux aussi bien que locaux interviennent dans le déclenchement des grosses avalanches de neige.

Au plan méthodologique, le fait que seulement trois avalanches aient été enregistrées dans les trois transects (tous indicateurs confondus) traduit la complémentarité des données d'un transect à l'autre et l'importance de bien couvrir l'ensemble de la zone d'arrivée des avalanches. Le nombre décroissant d'événements enregistrés depuis la partie inférieure vers la partie supérieure de la zone d'arrivée indique que la zone à proximité de la forêt épargnée par les coulées de neige présente le potentiel dendrochronologique le plus élevé. Ce potentiel diminue rapidement en montant dans le versant, car chaque nouvelle avalanche peut en effet contribuer à faire disparaître les traces d'avalanches anciennes.

\section{CONCLUSION}

Cette étude a permis de calculer la fréquence (ou probabilité) annuelle minimale des grosses avalanches de neige dans le couloir nord du mont Hog's Back depuis la fin du 
$\mathrm{XIX}^{\mathrm{e}}$ siècle. Aucune des grosses avalanches révélées par la dendrochronologie n'a atteint la base du versant depuis un siècle en raison du changement de l'inclinaison de la pente dans la zone d'arrivée et du rôle d'écran joué par la forêt mature sous les $450 \mathrm{~m}$ d'altitude. Compte tenu de la fréquence élevée des grosses avalanches depuis le milieu des années 1940, la protection de cette bande forestière contribuerait grandement à atténuer les risques que les grosses avalanches n'atteignent un jour les infrastructures sises au pied du versant (lignes de transport électrique et Route régionale 299 située en contrebas). Une meilleure compréhension des facteurs responsables du déclenchement des avalanches de neige en Gaspésie permettrait d'assurer un encadrement plus sécuritaire des sports de plein air en montagne, dont la popularité est en croissance.

\section{REMERCIEMENTS}

Nous remercions les personnes et organismes qui ont contribué à la préparation de cet article. Les commentaires et suggestions formulés par les évaluateurs de $G p Q$, MM. Bernard Denneler et Brian Luckman, complétés par les remarques de M. Pierre J.H. Richard, rédacteur en chef, ont grandement contribué à l'amélioration du manuscrit. Cette étude a été rendue possible grâce aux subventions obtenues par Louise Filion et Bernard Hétu du Fonds pour la formation des chercheurs et l'aide à la recherche (Fonds FCAR, Gouvernement du Québec) et du Conseil de recherches en sciences naturelles et génie du Canada (CRSNG). Dominic Boucher a bénéficié d'une bourse d'étude du CRSNG. Les figures ont été réalisées par Suzanne Gagnon. François Truchon a colligé et traité les données météorologiques de la station du Cap-Seize. Violaine Lafortune, Mathieu Pelletier, Stéphane Babin et feu Stéphane Beaudoin ont collaboré à la cueillette des données sur le terrain.

\section{RÉFÉRENCES}

ANENA (Association nationale pour l'étude de la neige et des avalanches) 1996. Guide neige et avalanches : connaissances, pratiques, sécurité. Édisud, Aix-en-Provence, $317 \mathrm{p}$.

Bégin, C. et Filion, L., 1985. Analyse dendrochronologique d'un glissement de terrain de la région du Lac à l'Eau Claire (Québec nordique). Canadian Journal of Earth Sciences, 22 : 175-182.

Boucher, D., 2000. Projet d'implantation d'un centre d'avalanche dans le parc de la Gaspésie. Municipalité régionale de comté de Denis-Riverin, SainteAnne-des-Monts, $26 \mathrm{p}$.

Brisebois, D. et Brun, J., 1994. La plate-forme du Saint-Laurent et les Appalaches, p. 95-117. In M. Hocq, édit., Géologie du Québec. Les Publications du Québec, MM94-01, Québec, 154 p.

Burrows, C.J. et Burrows, V.L., 1976. Procedures for the study of snow avalanche chronology using growth layers of woody plants. Institute of Arctic and Alpine Research, Boulder, Occasional Paper, 23, 54 p.

Butler, D.R. et Malanson, G.P., 1985a. A reconstruction of snow-avalanche characteristics in Montana, U.S.A., using vegetative indicators. Journal of Glaciology, 31 : 185-187.

1985b. A history of high-magnitude snow avalanches, southern Glacier National Park, Montana, U.S.A. Mountain Research and Development, 5 : 175-182.

Canadian Avalanche Association, 1995. Observation Guidelines and Recording Standards for Weather, Snowpack and Avalanches. Canadian Avalanche Association, Revelstoke, $98 \mathrm{p}$.
Carrara, P.E., 1979. The determination of snow avalanche frequency through tree-ring analysis and historical records at Ophir, Colorado. Geological Society of America Bulletin, 90 : 773-780.

Doyon, M. et Valiquette, G., 1991. Roches magmatiques du centre-nord de la Gaspésie. Ministère de l'Énergie et des Ressources, Québec, Rapport ET 90-03, 69 p.

Dubé, S., 1999. Impacts dendroécologiques et fréquence séculaire des avalanches sur trois versants boisés de la Gaspésie septentrionale, Québec. Mémoire de maîtrise, Département de géographie, Université Laval, 69 p.

Dubé, S., Filion, L. et Hétu, B., 2004. Tree-ring reconstruction of high-magnitude snow avalanches in the Northern Gaspé Peninsula, Québec. Arctic, Antarctic and Alpine Research, 36 : 541-550.

Gagnon, R.M., 1970. Climat des Chic-Chocs. Ministère des Richesses naturelles, Québec, MP 36, $103 \mathrm{p}$.

Gaumond, M. et Hamelin, L.-É., 1960. Note de périglaciaire comparé des monts Washington et Jacques-Cartier. Cahiers de Géographie de Québec, 7 : 217-218.

Girard, J.-F. et Hétu, B., 1989. Le Mont-Albert, Parc de la Gaspésie. Inventaire des avalanches et cartographie des zones à risques, Ministère du Loisir, de la Chasse et de la Pêche, Québec, $36 \mathrm{p}$.

1994. Les mouvements de masse en Gaspésie septentrionale. HydroQuébec, Rimouski, 45 p.

Gray, J.T. et Brown, R.J.E., 1979. Permafrost presence and distribution in the Chic-Chocs Mountains, Gaspésie, Québec. Géographie physique et Quaternaire, $33: 299-316$

1982. The influence of terrain factors on the distribution of permafrost bodies in the Chic-Chocs Mountains, Gaspésie, Québec, p. 23-25. In Proceedings of the 4th Canadian Permafrost Conference (Calgary, 2 au 6 mars 1981), National Research Council of Canada, Ottawa, 171 p.

Hétu, B. et Dubé, J.-F., 1995. L'avalanche meurtrière du 10 mars 1995 à BlancSablon (mont Bon-Enfant, Basse-Côte-Nord). Direction de la sécurité civile du Québec, Québec, 8 p.

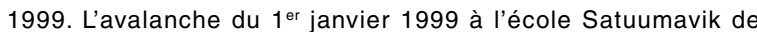
Kangiksualujjuak (Québec nordique). Direction de la sécurité civile du Québec, Québec, $15 \mathrm{p}$

Hétu, B. et Gray, J.T., 2000. Effects of environmental change on scree slope development throughout the postglacial period in the Chic-Choc Mountains in the northern Gaspé Peninsula, Québec. Geomorphology, 32 : 335-355.

Hétu, B. et Vandelac P., 1989. La dynamique des éboulis schisteux au cours de I'hiver, Gaspésie septentrionale, Québec. Géographie physique et Quaternaire, $43: 389-406$

Jamieson, B. et Brooks, G.R., 1998. Regional snow avalanche activity and known fatal avalanche accidents for Canada (1863 to June 1997). Geological Survey of Canada, Open File 3592, 1 carte, échelle approximative de 1/14 000000

Jamieson, B. et Geldsetzer, T., 1997. Avalanches au Canada, Volume 4 : 1984 1996. Canadian Avalanche Association, Revelstoke, 210 p.

Johnson, E.A., 1987. The relative importance of snow avalanche disturbance and thinning on canopy plant populations. Ecology, $68: 43-53$.

Johnson, E.A., Hogg, L. et Carlson, C.S., 1985. Snow avalanche frequency and velocity for the Kananaskis Valley in the Canadian Rockies. Cold Regions Science and Technology, $10: 141-151$

Larocque, S., Hétu, B. et Filion, L., 2001. Geomorphic and dendroecological impacts of slushflows in Central Gaspé Peninsula (Québec, Canada). Geografiska Annaler, 83A : 191-201.

McClung, D. et Schaerer, P.A., 1993. The Avalanche Handbook. The Mountaineers, Seattle, $271 \mathrm{p}$

Mears, A.I., 1992. Snow-avalanches hazard analysis for land-use planning and engineering. Colorado Geological Survey, Department of Natural Resources, Denver, Bulletin $49: 17-19$.

Niemann, K.O., 1982. Observations of snow avalanche activity in the Kananaskis region, Alberta. The Albertan Geographer, 18 : 29-42. 
Patten, R.S. et Knight, D.H., 1994. Snow avalanches and vegetation pattern in Cascade Canyon, Grand Teton National Park, Wyoming, U.S.A. Arctic and Alpine Research, $26: 35-41$.

Potter, N., Jr, 1969. Tree-ring dating of snow avalanche tracks and the geomorphic activity of avalanches, Northern Absaroka Mountains, Wyoming. Geological Society of America, Special Paper 123 : 141-165.

Rayback, S.A., 1998. A dendrogeomorphological analysis of snow avalanches in the Colorado Front Range, USA. Physical Geography, 19 :502-515.

Schaerer, P.A., 1987. Avalanche Accidents in Canada. III. A Selection of Case Histories 1978-1984. National Research Council of Canada, Ottawa, Institute for Research in Construction Paper 1468, 138 p.

Shroder, J.F., Jr., 1978. Dendrogeomorphological analysis of mass movement on Table Cliffs Plateau, Utah. Quaternary Research, $9:$ 168-185.
1980. Dendrogeomorphology: Review and new techniques of tree-ring dating. Progress in Physical Geography, 4 : 161-180.

Stethem, C.J. et Schaerer, P.A., 1979. Avalanche Accidents in Canada. I. A Selection of Case Histories of Accidents 1955 to 1976. National Research Council of Canada, Ottawa, Division of Building Research Paper 834, 114 p.

1980. Avalanche Accidents in Canada. II. A Selection of Case Histories of Accidents 1943 to 1978. National Research Council of Canada, Ottawa Division of Building Research Paper 926, $75 \mathrm{p}$

Veblen, T.T., Hadley, K.S., Nel, E.M., Kitzberger, T., Reid, M., et Villalba, R. 1994. Disturbance regime and disturbance interactions in a Rocky Mountain subalpine forest. Journal of Ecology, 82 : 125-135.

Veillette, J. et Cloutier, M., 1993. Géologie des formations en surface, Gaspésie, Québec. Commission géologique du Canada, Ottawa, carte 1804A, échelle de $1 / 250000$. 